\title{
Standard Deviation of Diameter
}

National Cancer Institute

\section{Source}

National Cancer Institute. Standard Deviation of Diameter. NCI Thesaurus. Code C120717.

A measure of the range of values in a set of values that describe the diameter of an entity. 